\title{
Acceleration of MCNP calculations for small pipes configurations by using Weigth Windows Importance cards created by the SN-3D ATTILA
}

\author{
Eric Castanier ${ }^{1, *}$, Loic Paterne ${ }^{1}$, and Céline Louis ${ }^{1}$ \\ ${ }^{1}$ AREVA PROJETS, 1 rue des hérons, 78182 Montigny le Bretonneux, France
}

\begin{abstract}
In the nuclear engineering, you have to manage time and precision. Especially in shielding design, you have to be more accurate and efficient to reduce cost (shielding thickness optimization), and for this, you use 3D codes. In this paper, we want to see if we can easily applicate the CADIS methods for design shielding of small pipes which go through large concrete walls. We assess the impact of the WW generated by the 3D-deterministic code ATTILA versus WW directly generated by MCNP (iterative and manual process). The comparison is based on the quality of the convergence (estimated relative error $(\sigma)$, Variance of Variance (VOV) and Figure of Merit (FOM)), on time (computer time + modelling) and on the implement for the engineer.
\end{abstract}

\section{Introduction}

In the nuclear engineering, you have to manage time and precision. Especially in shielding design, you have to be more accurate and efficient to reduce cost (shielding thickness optimization), and for this, you use 3D codes. On the other hand, run 3D Monte Carlo or deterministic codes requires time which is not always compatible with the projects dead line. So, to solve this dilemma, the Oak Ridge National Laboratory (ORNL) has developed two methods for increasing the efficiency of fixed-source MC simulations: The Consistent Adjoint Driven Importance Sampling (CADIS) method [1] and the Forward Weighted CADIS (FW-CADIS) method [2]. These methods utilize the results of approximate 3-D discrete ordinates transport calculations to generate consistent space- and energy-dependent source and transport (weight windows - WW) biasing parameters for MCNP code [3].

In this paper, we want to see if we can easily applicate these methods for design shielding for typical penetrations met in the French Fuel Cycle Re-processing plant located in La Hague (see figure 1).

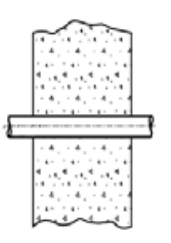

Restilinear penertration

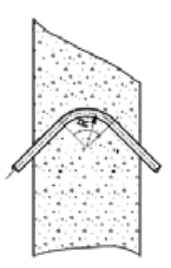

un $\mathrm{n}$ type penetration

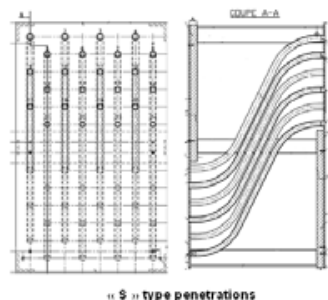

us in tope penectrations
Fig. 1. View of the typical penetrations.
For these penetrations, we need sometimes to design shielding to reduce the shielding defect in the wall due to these penetrations.

\section{Methods}

The goal of this study is to evaluate the impact of the WW generated by the 3D-deterministic code ATTILA [4] versus WW directly generated by MCNP (iterative and manual process - see figure 2 ).

In the iterative method; we chain several MCNP calculations where we use the weight window card created by the previous calculation.

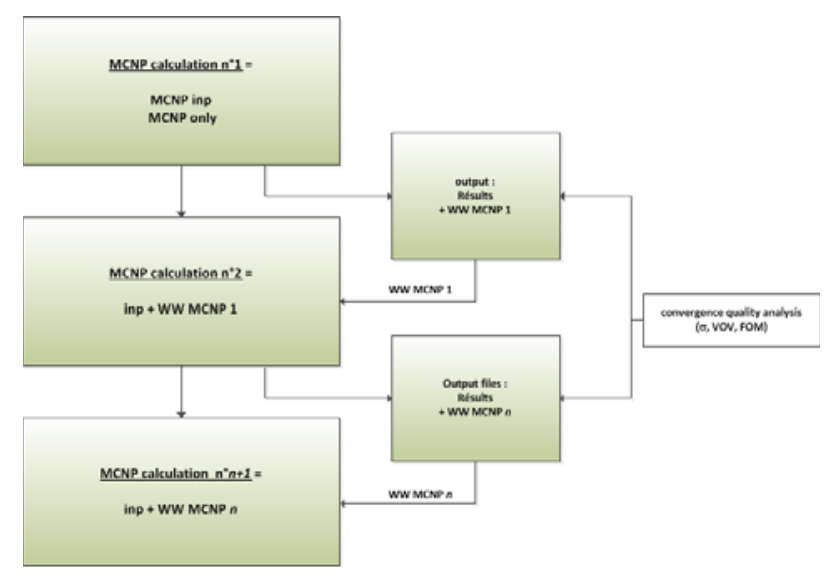

Fig. 2. Iterative method.

In the step 1, we create our first weight windows card. In the same time, we analyze the convergence quality and if it is not acceptable, we have to run a second calculation.

Corresponding author: eric.castanier@areva.com 
For this one, we use the weight window card created in the first calculation. We analyze the convergence quality of the result and so on to reach a good convergence.

With the CADIS method, we create the first weight window card by a 3-D discrete ordinates transport calculations (see figure 3).

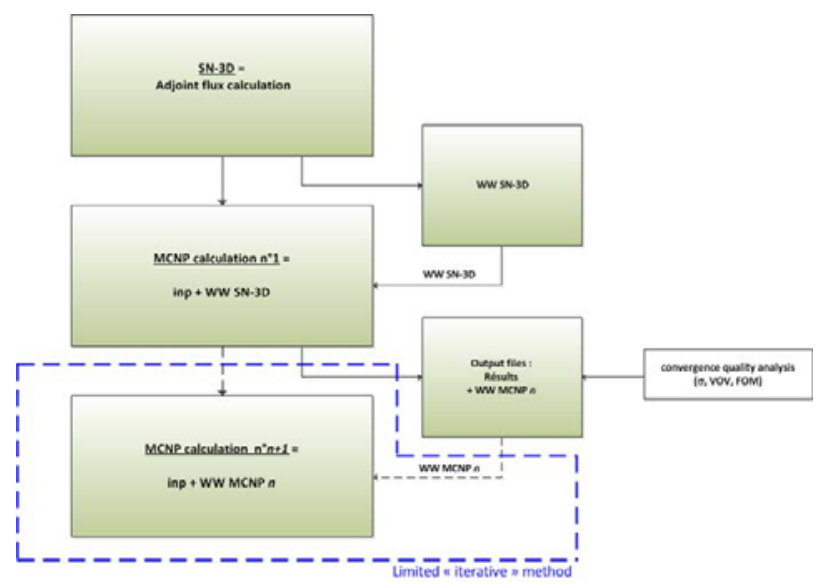

Fig. 3. CADIS method.

It allows to reduce significantly the MCNP iterative calculations.

The comparison is based on the quality of the convergence (estimated relative error $(\sigma)$, Variance of Variance (VOV) and Figure of Merit (FOM)), on time (computer time + modelling) and on the implement for the engineer.

\section{Calculations}

\subsection{Geometry}

The configuration is very simple (see fig. 2): an homogeneous cubic source of caesium-137 $(100 \mathrm{~cm} \mathrm{x}$ $100 \mathrm{~cm} \times 100 \mathrm{~cm}$ ) faces a pipe crossing a concrete $1 \mathrm{~m}-$ thick wall.

To discern the importance of the different parameters of the deterministic code (SN order, mesh...), 3 diameters for pipes are considered: $1 \mathrm{~cm}, 5 \mathrm{~cm}$ and $10 \mathrm{~cm}$.

Table 1. Source characteristics.

\begin{tabular}{|c|c|c|}
\hline Iso & $\mathbf{E}(\mathbf{M e V})$ & $\mathbf{G a m m a} / \mathbf{s}$ \\
\hline Cs137 & 0.662 & 0.85 \\
\hline
\end{tabular}

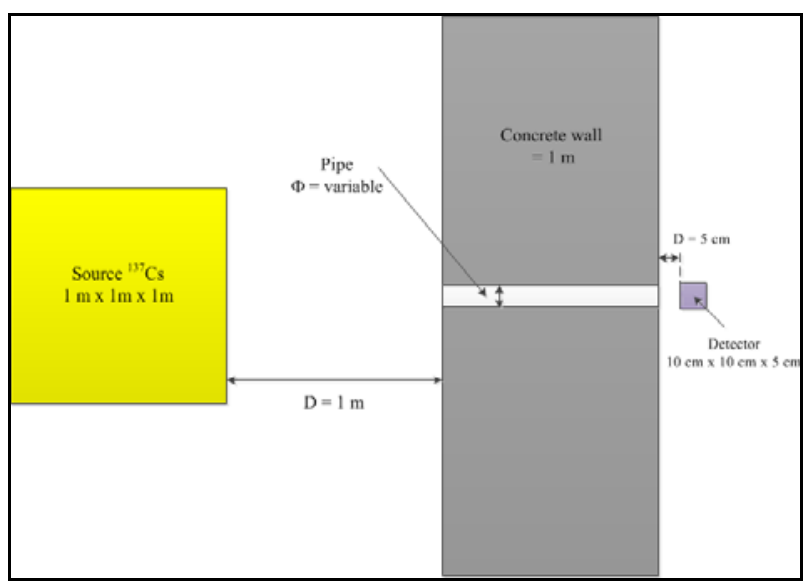

Fig. 4. View of the geometric configuration.

\subsection{Codes}

Dose Equivalent Rates (DER) due to gamma photons are calculated with the MCNP code, version 4C2 [3]. MCNP is a continuous-energy transport code for simulating nuclear processes based on the Monte Carlo method (stochastic method). It enables the transport of particles (neutrons, photons, electrons...) through the matter and simulates their interactions by means of probability laws. As the method is based on a stochastic method, the results go along with a statistical error. The upper limit criterion of this error is set to $10 \%$.

The DER is obtained with the "Track length estimate of cell flux", called F4 tally, in a parallelepiped cell $(10 \mathrm{~cm} \times 10 \mathrm{~cm} \times 5 \mathrm{~cm})$.

The 3-D discrete ordinates transport code used to generate consistent space- and energy-dependent source and transport (weight windows) is ATTILA 8.0 code [4]. ATTILA 8.0 is a three dimensional (3-D) $S_{n}$ transport theory code. Attila solves the linearized Boltzmann transport equation in first order form using a tri-linear discontinuous spatial differencing on an arbitrary tetrahedral mesh. Attila comes with a graphical user interface (GUI) which allows direct integration with CAD software programs such as Solidworks ${ }^{\circledR}$.

Applied flux to dose conversion coefficients are extracted from ICRP 74 [5].

\subsection{WW generation}

The mesh parameters fixed to realize the WW generation by ATTILA are shown on table 2 . 
Table 2. Mesh dimensions.

\begin{tabular}{|c|c|c|c|}
\hline & \multicolumn{3}{|c|}{ Mesh sizing (cm) } \\
\hline \multirow{2}{*}{$\begin{array}{c}\text { Pipe } \\
\text { diameter }\end{array}$} & Wall & Source & Global mesh \\
\hline $1 \mathrm{~cm}$ & \multirow{2}{*}{6} & 5 & \multirow{2}{*}{40} \\
\cline { 1 - 1 } $5 \mathrm{~cm}$ & \multirow{2}{*}{6} & 6 & \\
\cline { 3 - 3 } $10 \mathrm{~cm}$ & & 7 & \\
\cline { 3 - 3 } & & \multicolumn{2}{|c|}{} \\
\hline
\end{tabular}

Calculations are performed with a triangular Chebychev Legendre quadrature and an Sn order of 24 to 42 .

A gamma cross section library based on RADION15 XS library with a specific group structure (34 groups, $11 \mathrm{MeV}-1 \mathrm{E}-3 \mathrm{MeV}$ ) is used.

Unfortunately, we have no choice but to define a uniform WW mesh with ATTILA, while we can optimize it with the WW generator from MCNP (see figure 5).

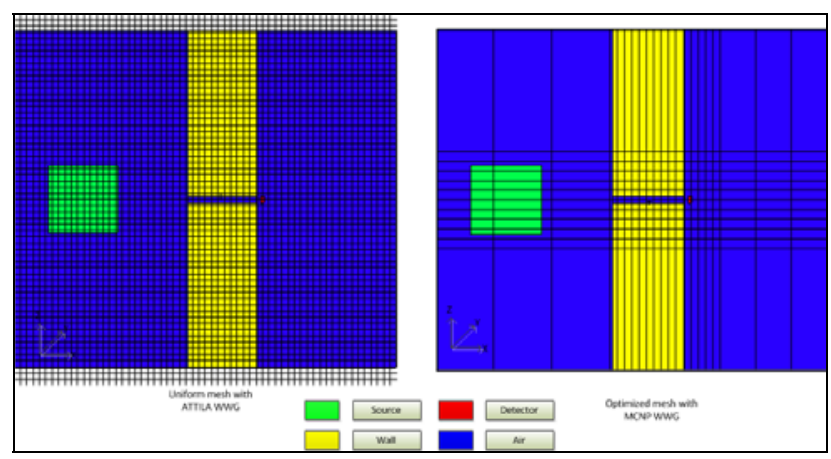

Figure 5. Mesh used to generate WW with ATTILA (left side) and MCNP (right side).

\section{Results}

\subsection{Adjoint flux}

One example of the adjoint flux is shown on the figure 6.

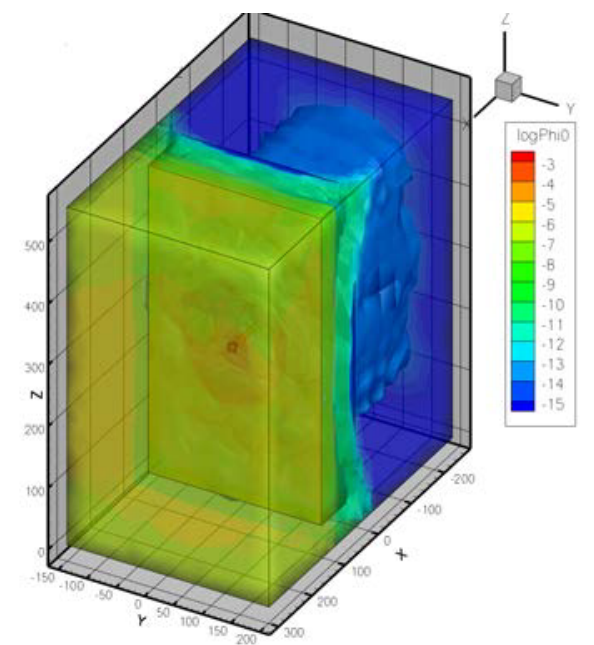

Figure 6. View of the adjoint flux

\subsection{WW card}

One example of the WW card generated either by ATTILA or by MCNP is shown on the figure 7.

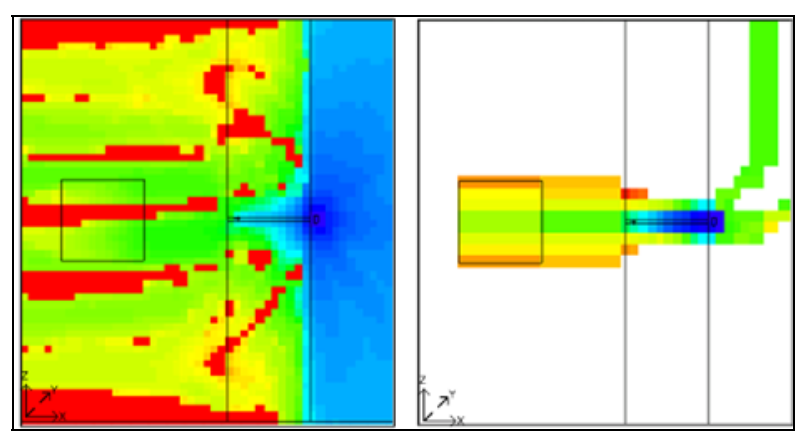

Figure 7. WW card generated by ATTILA (left side) and MCNP (right side)

The particles which go from yellow/green region to dark blue region will be facilitated (and so well sampled) while the ones which go to the orange will be killed rapidly. We can see on the map created by ATTILA some non-well defined regions (in red) due to negative flux.

On the map created by ATTILA, we can see in red some non-well defined regions due to negative flux. If there are too many regions with negative flux, especially around the regions of interest, you will generate a bad weight window and you will have many problems with the MCNP calculation.

\subsection{Results - discussion}

For rectilinear pipes with large diameter $(\Phi>10 \mathrm{~cm})$, the WW generated by ATTILA is not efficient: it consumes time and provides no more information than a simple MCNP calculation with using usual convergence technics.

For rectilinear pipes with smaller diameter $(\Phi \leq 5 \mathrm{~cm})$, we had to increase the SN order ( 24 to 42 due to negative flux). The ATTILA's meshing and the normalization are significant on the final MCNP DER's value. It is important to be very careful on the ATTILA calculation to be sure that it generates a good WW card. In any case, we found it necessary to create a new WW card with MCNP to make sure to get a consistent result.

\subsection{Future}

The ATTILA 9.0 code was released at the beginning of spring. It offers new features like direct MCNP6 calculations. We could also define region specific mesh like in the MCNP weight windows generator. The next step will be to see if our results are more consistent with these new features. 


\section{References}

1. J.C. Wagner, A. Haghighat, Nucl. Sci. 128, 186 (1998)

2. J.C. Wagner, D.E. Peplow, S.W. Mosher, Nucl. Sci. 176, 37 (2014)

3. MONTE CARLO TEAM, "MCNP-A General Monte Carlo N-Particle Transport Code, Version 4c" LA -13709-M, Los Alamos National Laboratory (2000)

4. J.M. McGhee, T.A. Wareing and D.A. Barnett Jr, Attila User's Manual, Transpire Inc. (2007)

5. ICRP publication 74, Conversion Coefficients for use in Radiological Protection against External Radiation, Annals of the ICRP 26(3/4) (1996) 